치위생(학)과 학생의 셀프 리더십이 임상실습 스트레스와 스트레스 대처방식에 미치는 영향

-대구 경북 중심으로-

최규일 · 윤현경 ${ }^{1}$

충청대학교 치위생과 $\cdot{ }^{1}$ 안동과학대학교 치위생과

\title{
Effect of self-leadership of students majoring in dental hygiene on clinical practice-induced stress and countermeasures against the stress
}

\section{- focusing on Daegu and Gyeongbuk -}

Gyu-Yil Choi · Hyun-Kyung Yun ${ }^{1}$

Department of Dental Hygiene, Chungcheong College $\cdot{ }^{1}$ Department of Dental Hygiene, Andong Science College

Received : 2 July, 2013
Revised : 30 September, 2013
Accepted : 10 October, 2013
Corresponding Author
Hyun-Kyung Yun
Department of Dental Hygiene, Andong
Science College
189, Seoseongil, Seohu, Andong
760-709, Korea.
Tel : + 82-54-851-3663
$\quad+82-10-9936-8321$
Fax : + 82-54-852-9907
E-mail : yhk8321@hanmail.net

\section{서론}

현대의 조직은 산업사회에서 지식정보화 사회로, 집중화에 서 탈 집중화 사회로 가는 과정 가운데, 그 어느 때보다 경쟁이 치열해지고 있으며, 또한 그 어느 때보다 조직을 실제로 이끌

\section{ABSTRACT}

Objectives : This study aims to investigate the relationship between the self-leadership and stress of students majoring in dental hygiene in clinical practice. This study will be helpful to cope with clinical practice effectively.

Methods : Subjects were 221 dental hygiene students from 4 universities in Daegu and Gyeongbuk. Data were abalyzed by independent t-test and multiple regression analysis.

Results : Stress-response was 60.36 points in high self-leadership group and 57.20 points in kow self-leadership group. It was statistically significant. Clinical practice-induced stress was made by self-expectation, rehearsal, and self-criticism. Stress was able to be overcome by self-reward and positive mind.

Conclusions : It is necessary to take the curriculum into consideration which can improve the self-leadership before the clinical practice or at the early phase of clinical practice in order to ensure that the students majoring in dental hygiene are exposed to less stress arising from clinical practice and better cope with such stress.

Key Words : clinical practice-Induced stress, countermeasures against the stress, self-leadership 색인 : 셀프리더십, 스트레스 대처방식, 임상실습 스트레스 
식과 새로운 기술뿐만 아니라 높은 수준의 자율성과 독립성 을 갖고 조직에 대한 강한 일체감과 적극적인 참여를 유발 할 수 있는 새로운 리더십이 요구되고 있달. 이와 같이 리더 십을 바라보는 관점도 이 시대에 맞는 새로운 패러다임으로 의 변화가 필요하게 되었고 그 결과 기존의 타율적인 성향에 서의 리더십이 아닌, 자기 자신으로부터 리더십을 발휘하도 록 하는 인간 내부의 기본적인 성향인 '자율성'을 강조한 새로 운 관점의 셀프리더십이 등장하게 되었다 ${ }^{3)}$. 셀프리더십에 의하면 조직구성원의 자아관리 역량과 내적인 동기부여에 초점을 맞춤으로써 개인이 창의성과 자발적인 능력발휘를 통해 조직의 성과를 높인다고 하였다 ${ }^{4)}$.

최근 의료환경의 급속한 변화를 겪으면서 치과위생사의 전 문성 영역 확대에 대한 욕구가 증가함에 따라 치과위생사들 의 리더십은 필수적인 요소로 요구되고 있으며 특히 셀프리 더십의 중요성이 부각되고 있다) . 변화가 빠르고 불확실한 환경은 일반적으로 스트레스를 유발하는 상황으로 이런 상황 에 처한 개인은 자신이 지니고 있는 특성에 따라 다양한 대처 방식을 보일 수 있닿. 셀프리더십은 예비 치과위생사인 치 위생과 학생들에게도 필수적인 것으로 교육과정에 개설, 운 영이 필요하다고 사료된담) . 그러나 임상실습 현장에서 치 위생(학)과 학생들에게 능동성, 자율성, 책임성 등을 요구 하 는데 대학입시로 인해 수동적, 의존적인 생활방식에 길들여 져 있어 실습 현장의 요구에 부합하는데 어려움을 겪을 뿐만 아니라 임상의 새로운 환경과 예상치 못하는 위기상황, 대인 관계, 역할수행 등으로 인한 스트레스를 경험하게 된다. 이러 한 스트레스가 지속되면 실습에 적응하지 못할 뿐만 아니라
직업선택과 전문성에도 영향을 미칠 수 있다 ${ }^{7}$. 이러한 스트 레스 상황에서 적극적인 대처보다 소극적인 대처 방식을 많 이 사용하는데 치위생(학)과 학생들에게 임상실습 스트레스 를 줄여주고 효과적인 대처를 할 수 있는 임상실습 교육 방안 은 반드시 필요할 것이다. 따라서 자기 스스로 합리적인 방향 으로 이끌어나가고 자율성과 책임성을 강조하는 셀프리더십 과 임상실습 스트레스와 대처방식의 관계를 알아보는 것은 의미가 있다고 할 수 있겠다 ${ }^{8)}$. 보건직종의 셀프리더십과 임 상실습스트레스 및 대처방식의 상관성에 관한 연구는 보고되 고 있고 ${ }^{5,8)}$, 치위생(학)과를 대상으로 한 연구는 셀프리더십이 스트레스 대처방식에 미치는 영향에 관한 연구도 보고되고 있지만 아직까지는 미흡한 실정이다).

이에 본 연구는 치위생(학)과 학생들의 셀프리더십과 임상 실습 스트레스 및 스트레스 대처방식의 관계에 대해 알아보 고 임상실습을 효과적으로 대처할 수 있는 방안을 마련하는 데 필요한 기초자료를 제공하고자 한다.

\section{연구대상 및 방법}

\section{1. 연구대상}

본 연구의 연구대상은 대구 경북에 소재한 4 개 대학교의 치위생(학)과에 재학 중인 2.3학년 총 221명을 대상으로 하였 으며 일반적 특징은 〈Table 1〉과 같다. 성별은 여자 218명 (98.6\%)였으며, 남자 3명(1.4\%)이었다. 종교는 없는 경우가 149 명(67.4\%)였으며 건강상태는 건강한 편이 114 명(51.6\%)

Table 1. The characteristics of the study subjects

\begin{tabular}{|c|c|c|c|}
\hline & & $\mathrm{N}$ & $\%$ \\
\hline \multirow{2}{*}{ Sex } & Female & 218 & 98.6 \\
\hline & Male & 3 & 1.4 \\
\hline \multirow{2}{*}{ Religion } & Yes & 72 & 32.6 \\
\hline & No & 149 & 67.4 \\
\hline \multirow{3}{*}{ Health } & Good & 114 & 51.6 \\
\hline & Usually & 96 & 43.4 \\
\hline & Bad & 11 & 5.0 \\
\hline \multirow{4}{*}{ Interpersonal } & Not good & 4 & 1.8 \\
\hline & Usually & 97 & 43.9 \\
\hline & Good & 91 & 41.2 \\
\hline & Very good & 29 & 13.1 \\
\hline \multirow{6}{*}{ Entry motivation } & Grades & 12 & 5.4 \\
\hline & Recommended around & 55 & 24.9 \\
\hline & Easy employment & 69 & 31.2 \\
\hline & Aptitudes and interests & 15 & 6.8 \\
\hline & Expertise & 65 & 29.4 \\
\hline & Others & 5 & 2.3 \\
\hline Total & & 221 & 100.0 \\
\hline
\end{tabular}


Table 2. Self-leadership, clinical practice-induced stress, confidence level of the stress measurement tool

\begin{tabular}{llcc}
\hline & Sub-variables & No. of questions & Confidence level \\
\hline & Self-expectation & 3 & 0.82 \\
& Rehearsal & 3 & 0.70 \\
& Goal-setting & 3 & 0.69 \\
\multirow{3}{*}{ Self-leadership } & Self-reward & 3 & 0.66 \\
& Self-criticism & 3 & 0.70 \\
& Constructive idea & 3 & 0.69 \\
& Total & 18 & 0.84 \\
\hline Clinical practice-induced stress & 12 & 0.90 \\
\hline Countermeasures against stress & 24 & 0.84 \\
\hline
\end{tabular}

였으며 대인관계는 보통과 좋음이 각각 97 명, 91명으로 $43.9 \%, 41.2 \%$ 였다.

\section{2. 연구도구}

\section{1 셀프리더십}

셀프리더십을 측정하기 위해 본 연구에서는 $\mathrm{Manz}^{9}$ 가 개 발한 설문지를 기초로 김 ${ }^{10}$ 과 박 ${ }^{8}$ 의 수정 - 보완한 도구를 사용하였다. 본 도구는 자기기대, 리허설, 목표설정, 자기보 상, 자기비판, 건설적 사고 등 6 개 요인으로 각각 3 문항씩 총 18 문항으로 구성되어 있다. 측정 기준은 5점 척도로써 '항 상 그렿다' 5 점에서 '전혀 그렇지 않다' 1 점의 범위에 있으며 점수가 높을수록 셀프리더십이 높음을 의미한다. 김이 의 연 구에서 도구의 신뢰도 Cronbach's $\alpha$ 는 0.87 , 박' ${ }^{8}$ 연구에서의 Cronbach's $\alpha$ 는 0.77 이었고 본 연구에서는 Cronbach's $\alpha$ 0.84 로 나타났다.

\section{2. 임상스트레스}

임상실습 스트레스을 측정하기 위해 심 ${ }^{11)}$ 과 황 ${ }^{12}$ 의 연구 도구를 참고하였고, 일반적 스트레스 량 측정을 위해서는 우 리나라의 실정에 맞게 개발한 간이 스트레스 측정도구
(BEPSI) 배 등흐를 사용하였다. 환경, 대인관계, 역할 및 활동, 이상과 가치 등 요인별 스트레스 관련사항, 진료 분야별 스트 레스 정도, BEPSI의 문항으로 구성되었다. 임상실습 시 겪는 요인별 스트레스는 5점 척도를 사용하였고, '전혀 느끼지 않는다' 1 점 '거의 느끼지 않는다' 2점 '약간 느낀다' 3점 '심하 게 느낀다' 4 점 '매우 심하게 느낀다' 5 점으로 측정하여 점수가 높을수록 스트레스가 높음을 의미한다. 본 연구에서는 도구 신뢰도 Cronbach's $\alpha$ 는 0.90로 나타났다.

\section{3. 스트레스 대처방식}

스트레스 대처방식을 측정하기 위하여 본 연구에서는 Folkman과 Lazarus ${ }^{14}$ 가 개발한 스트레스 대처방식의 척도

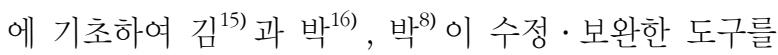
사용하였다.

본 척도는 4점 척도로 '전혀 사용하지 않음' 1 점에서 '매우 많이 사용' 4점까지 점수가 높을수록 스트레스 대처방식을 많이 사용한다는 것을 의미한다. 박 ${ }^{16)}$ 의 연구에서 신뢰도 Cronbach's $\alpha$ 는 0.81 , 박 ${ }^{8}$ 의 연구에서는 Cronbach's $\alpha$ 는 0.77 이었고, 본 연구에서는 스트레스 대처방식 문항에 대한 Cronbach's $\alpha$ 는 0.84 로 나타났다〈Table 2〉.

Table 3. Mean and standard deviation of the upper rank group and lower rank group of self-leadership \& statistical analysis of the difference

\begin{tabular}{|c|c|c|c|c|c|c|}
\hline Sub-variables & Group & $\mathrm{N}$ & $\mathrm{M}$ & SD & $t$ & $p$ \\
\hline \multirow{2}{*}{ Self-expectation } & High group $>10$ & 106 & 12.03 & 1.175 & \multirow{2}{*}{18.257} & \multirow{2}{*}{$\langle 0.001$} \\
\hline & Low group $\leq 10$ & 115 & 8.9 & 1.36 & & \\
\hline \multirow{2}{*}{ Rehearsal } & High group $>10$ & 92 & 12.0978 & 1.20493 & \multirow{2}{*}{18.219} & \multirow{2}{*}{$<0.001$} \\
\hline & Low group $\leq 10$ & 129 & 8.6202 & 1.52169 & & \\
\hline \multirow{2}{*}{ Goal-setting } & High group $>10$ & 105 & 12.0381 & 1.15961 & \multirow{2}{*}{18.984} & \multirow{2}{*}{$<0.001$} \\
\hline & Low group $\leq 10$ & 116 & 8.819 & 1.34228 & & \\
\hline \multirow{2}{*}{ Self-reward } & High group $>11$ & 109 & 12.945 & 1.05264 & \multirow{2}{*}{18.275} & \multirow{2}{*}{$\langle 0.001$} \\
\hline & Low group $\leq 11$ & 112 & 9.7679 & 1.48859 & & \\
\hline \multirow{2}{*}{ Self-criticism } & High group $>9$ & 103 & 11.0874 & 1.19727 & \multirow{2}{*}{17.443} & \multirow{2}{*}{$<0.001$} \\
\hline & Low group $\leq 9$ & 118 & 8.0254 & 1.38652 & & \\
\hline \multirow{2}{*}{ Constructive idea } & High group $>9$ & 117 & 11.08 & 1.196 & \multirow{2}{*}{14.828} & \multirow{2}{*}{$<0.001$} \\
\hline & Low group $\leq 9$ & 104 & 8.09 & 1.174 & & \\
\hline
\end{tabular}


Table 4. Difference in the level of clinical practice-induced stress \& the countermeasures against stress, depending on the extent of self-leadership

\begin{tabular}{clcrrrr}
\hline Sub-variables & \multicolumn{1}{c}{ Group } & N & M & SD & t & p \\
\hline \multirow{2}{*}{ Clinical practice-induced stress } & High group self-leadership & 100 & 116.94 & 25.19 & \multirow{2}{*}{-1.676} & \multirow{2}{*}{0.095} \\
& Low group self-leadership & 121 & 121.92 & 19.00 & & \multirow{2}{*}{2.383} \\
\multirow{2}{*}{ Countermeasures against stress } & High group self-leadership & 100 & 60.36 & 10.66 & \\
& Low group self-leadership & 121 & 57.20 & 9.00 & \\
\hline
\end{tabular}

Table 5. Correlation among subfactors of self-leadership ${ }^{\dagger}$

\begin{tabular}{|c|c|c|c|c|c|c|}
\hline & 1 & 2 & 3 & 4 & 5 & 6 \\
\hline Self-expectation & 1 & & & & & \\
\hline Rehearsal & $.482^{* *}$ & 1 & & & & \\
\hline Goal-setting & $.484^{* *}$ & $.430^{* *}$ & 1 & & & \\
\hline Self-reward & $.558^{* *}$ & $.462^{* *}$ & $.529^{* *}$ & 1 & & \\
\hline Self-criticism & $.181^{*}$ & $.342^{* *}$ & $.236^{*}$ & $.296^{*}$ & 1 & \\
\hline Constructive idea & $.480^{* *}$ & $.380^{* *}$ & .462 & $.327^{* *}$ & $.155^{*}$ & 1 \\
\hline
\end{tabular}

$p<.01$

†1.Rehearsal, 2.Rehearsal, 3.Goal-setting, 4.Self-reward, 5.Self-criticism, 6.Constructive idea

\section{3. 자료분석}

치위생과 학생의 셀프 리더십이 임상실습스트레스와 스트 레스 대처방안에 미치는 영향을 알아보기 위해서 치위생과의 셀프리더십이 평균점수보다 높은 경우를 상위집단으로 평균 점수보다 낮은 경우는 하위집단으로 설정하였다. 〈Table 3〉 과 같이 분리점(cut point)을 사이로 두 집단은 셀프 리더십의 하위요소에서 모두 유의한 차이를 보여 평균점을 사이에 두 고 상위집단과 하위집단이 이질집단임을 검증하였다. 자료 분석을 위한 도구는 SPSS 18.0을 사용하여 독립변수 t검정을 각각 실시하였다. 또한 셀프 리더십의 하위요소들이 치위생 과 학생들의 임상실습스트레스와 스트레스 대처정도를 설명 하고 예측하기 위해서 중다 회귀분석을 실시하였다.

\section{연구성적}

\section{1. 셀프 리더십에 따른 임상실습 스트레스와 스트레스 대처방안의 차이}

셀프 리더십이 높게 나타난 집단의 임상실습스트레스 평균 은 116.94였고, 셀프리더십이 낮게 나타난 집단의 임상실습 스트레스는 121.92 로 나타났고 통계적으로 유의미한 차이는
없었다. 셀프리더십 높은 집단의 스트레스 대처는 평균 60.36 이였고, 셀프리더십 낮은 집단의 평균은 57.20이었으며 두 집단은 통계적으로 유의한 것으로 나타났다〈Table 4〉.

\section{2. 셀프 리더십이 임상실습스트레스와 스트레스 대처방안에 미치는 영향}

\section{1. 셀프 리더십 하위요소간의 상관관계}

셀프 리더십의 하위요소간의 상관분석을 실시한 결과는 〈Table 5〉와 같다. 셀프 리더십 하위 요소 간에는 양의 상관 관계가 나타났다.

\section{2. 셀프 리더십과 임상실습스트레스와 스트레스 대처척도와의 관계}

셀프 리더십과 임상실습스트레스, 스트레스 대처척도와의 관계를 알아보기 상관분석을 실시한 결과는 〈Table 6)와 같 다. 셀프 리더십과 스트레스 대처척도간에 양의 상관관계가 나타났다.

\section{3. 셀프 리더십이 임상실습스트레스에 미치는 영향}

셀프 리더십의 하위요소중 임상실습스트레스를 예측할 수 있는 요소는 자기기대, 리허설, 자기비판으로 나타났으며, 목

Table 6. Relationship among self-leadership. clinical practice-induced stress, and scale of response coping with the stress

\begin{tabular}{lccc}
\hline & 1 & 2 & 3 \\
\hline Self-leadership & 1 & & \\
Clinical practice-induced stress & -0.041 & 1 & \\
Countermeasures against stress & $.243^{* *}$ & -0.022 & 1 \\
\hline
\end{tabular}
$\mathrm{p}\langle .01$ 
Table 7. Effect of self-leadership on clinical practice-induced stress

\begin{tabular}{|c|c|c|c|c|c|c|}
\hline & & \multicolumn{2}{|c|}{$\begin{array}{l}\text { Non-standardized } \\
\text { coefficients }\end{array}$} & \multirow{2}{*}{$\begin{array}{c}\begin{array}{c}\text { Standardized } \\
\text { coefficients }\end{array} \\
\beta \\
\end{array}$} & \multirow[t]{2}{*}{$t$} & \multirow[t]{2}{*}{$p$} \\
\hline & & B & S.E & & & \\
\hline \multirow{10}{*}{1} & Constant & 42.128 & 4.485 & & 9.394 & 0.000 \\
\hline & Self-expectation & -1.288 & 0.421 & -0.27 & -3.057 & $<0.01$ \\
\hline & Rehearsal & 0.763 & 0.356 & 0.176 & 2.145 & $<0.05$ \\
\hline & Goal-setting & -0.041 & 0.399 & -0.009 & -0.103 & 0.918 \\
\hline & Self-reward & 0.345 & 0.41 & 0.074 & 0.843 & 0.400 \\
\hline & Self-criticism & -0.668 & 0.301 & -0.157 & -2.218 & $<0.05$ \\
\hline & Constructive idea & -0.304 & 0.379 & -0.063 & -0.802 & 0.423 \\
\hline & $\mathrm{F}$ & 3.155 & & & & $<0.01$ \\
\hline & $R^{2}$ & 0.081 & & & & \\
\hline & Adjusted $R^{2}$ & 0.056 & & & & \\
\hline
\end{tabular}

표설정, 자기보상, 건설적 사고는 유의한 설명력을 보이지 않았다.

셀프 리더십의 하위요소가 임상실습스트레스와의 관계를 설명 하기 위한 회귀방정식은 Y' (임상실습스트레스) $=3.155-1.288 X^{\prime}$ (자기기대)이며, Y' (임상실습스트레스) $=3.155+.763 X^{\prime}$ (리허설), $Y^{\prime}\left(\right.$ 임상실습스트레스) $=3.155-0.668 X^{\prime}$ (자기비판)이며, 리더십 발휘 설명력은 8.1\%(수정된R제곱=.056)이다〈Table 7〉.

\section{4. 셀프 리더십이 스트레스 대처방안에 미치는 영향}

셀프 리더십의 하위 요소 중 스트레스 방안을 예측할 수 있는 요소는 자기보상과 건설적 사고로 나타났으며, 자기기 대, 리허설, 목표설정, 자기비판은 유의한 설명력을 보이지 않았다.

셀프 리더십의 하위요소가 스트레스 대처와의 관계를 설명 하기 위한 회귀방정식은 $Y^{\prime}($ 스트레스 대처 $)=4.419+1.1688 X^{\prime}$ (자기보상)이며, $Y^{\prime}($ 스트레스 대처 $)=4.419+1.163 X^{\prime}$ (건설적 사고)이며, 리더십 발휘 설명력은 $11 \%$ (수정된R제곱=.085이
다 〈Table 8$\rangle$.

\section{총괄 및 고안}

치위생(학)과 학생들에게 임상실습은 다양한 측면에서 스 트레스를 제공하고 있닥) 이러한 임상실습 관련 스트레스 는 임상실습에 대한 흥미와 요구 및 인내력을 감소시킴은 물론 학습저하를 야기한다 ${ }^{18}$.

임상실습 스트레스를 감소시키고 스트레스에 대처할 수 있 도록 하기 위해서는 개인적 특성이 고려되어져야 한다고 본 다 ${ }^{19)}$. 이를 위해 Neck 등이은 자기스스로에게 동기를 부여하 고 자신의 영향력을 행사하는 과정을 생각하는 셀프 리더십 함양을 제안한다.

셀프 리더십 자신들의 생활을 스스로 통제할 수 있는 자율 권이나 책임이 주어지는 것이고 셀프 리더십을 향상시키는 궁극적인 목적은 자신이 자신을 스스로 통제하는 것이라고 했다 $^{21)}$. 이에 대해 Manz와 $\mathrm{Sims}^{3)}$ 는 셀프리더십은 자기 스스

Table 8. Effect of self-leadership on countermeasures against stress

\begin{tabular}{|c|c|c|c|c|c|c|}
\hline & & \multicolumn{2}{|c|}{$\begin{array}{l}\text { Non-standardized } \\
\text { coefficients }\end{array}$} & \multirow{2}{*}{$\begin{array}{c}\text { Standardized } \\
\text { coefficients }\end{array}$} & \multirow[t]{2}{*}{$\mathrm{t}$} & \multirow[t]{2}{*}{$\mathrm{p}$} \\
\hline & & $\mathrm{B}$ & S.E & & & \\
\hline \multirow{10}{*}{1} & Constant & 39.851 & 4.54 & & 8.778 & 0.000 \\
\hline & Self-expectation & -0.181 & 0.427 & -0.037 & -0.425 & 0.671 \\
\hline & Rehearsal & -0.512 & 0.36 & -0.114 & -1.421 & 0.157 \\
\hline & Goal-setting & -0.099 & 0.404 & -0.02 & -0.244 & 0.807 \\
\hline & Self-reward & 1.168 & 0.415 & 0.242 & 2.815 & $<0.01$ \\
\hline & Self-criticism & 0.276 & 0.305 & 0.063 & 0.905 & 0.366 \\
\hline & Constructive idea & 1.163 & 0.383 & 0.236 & 3.034 & $<0.01$ \\
\hline & $\mathrm{F}$ & 4.419 & & \multicolumn{2}{|r|}{$<0.00$} & \\
\hline & $R^{2}$ & 0.11 & & & & \\
\hline & Adjusted $R^{2}$ & 0.08 & & & & \\
\hline
\end{tabular}


로 성취목표를 설정하고 그 목표 달성에 대한 보상을 스스로 정하는 등 자율성을 강조한 것이라고 하였다.

이러한 자기 스스로를 합리적인 방향으로 이끌어 가고 자 율과 책임을 강조하는 셀프 리더십과 임상 실습 스트레스와 스트레스 대처 방식의 관계를 알아보는 것은 의미가 있다고 하겠다 ${ }^{8)}$. 따라서 본 연구는 치위생과 학생들의 셀프리더십 에 따른 임상실습 스트레스와 스트레스 대처 방식을 알아보 고 결과를 토대로 대학시절 동안 셀프리더십 향상과 효과적 인 스트레스 대처를 도와줄 프로그램 개발을 위한 기초 자료 를 마련하고자 한다.

먼저 셀프 리더십에 따른 임상실습 스트레스와 스트레스 대처방안의 차이의 결과 셀프 리더십이 높게 나타난 집단의 임상실습스트레스 평균은 116.94였고, 셀프리더십이 낮게 나 타난 집단의 임상실습스트레스는 121.92 로 나타나 셀프리더 십이 높은 경우 임상실습 스트레스가 낮은 것으로 나타났다. 셀프리더십 높은 집단의 스트레스 대처는 평균 60.36이였고, 셀프리더십 낮은 집단의 평균은 57.20 이었으며 두 집단은 통 계적으로 유의한 것으로 나타나 셀프리더십이 높은 집단의 스트레스 대처를 잘 하는 것으로 나타났다. 이는 셀프리더십 임상실습 스트레스를 줄여주고 스트레스에 잘 대처할 수 있 는 방안이라고 제시한 박 ${ }^{8}$ 의 연구와도 일치된다. 양과 문 ${ }^{19}$ 의 연구에서는 간호대학생도 셀프리더십, 임상실습 만족도에 서 통계적인 유의한 정의 관계로 나타났다.

셀프 리더십이 높을수록 스트레스 대처에서 보다 적극적인 대처를 많이 사용하는 것을 알 수 있다. 보다 구체적으로 셀프 리더십과 임상실습 스트레스와 스트레스 대처 방안과는 상관이 있는 것으로 나타났는데 리더십 발휘 설명력 각각 $8.1 \%, 11 \%$ 로 나타났다.

양과 문 ${ }^{19}$ 의 연구에서도 간호대학생의 셀프리더십과 임상 실습 스트레스 및 임상실습 만족도 간의 상관성은 셀프리더 십과 임상실습 만족도에서 통계적으로 유의한 정의 관계로 나타났다. 뿐만 아니라 서문 등리 의 연구에서는 셀프리더십 은 업무성과에서도 밀접한 관계가 있는 것으로 나타나 셀프 리더십은 내적 동기부여에 초점을 맞춘 새로운 리더십 패러 다임으로써 셀프리더십을 위한 교육이나 프로그램 개발이 요구된다고 제안하고 있다.

또한 셀프 리더십의 하위 요소 중 임상실습 스트레스를 예 측할 수 있는 요소는 자기기대, 리허설, 자기비판으로 나타났 으며, 셀프 리더십의 하위 요소 중 스트레스 대처 방안을 예측 할 수 있는 요소는 자기보상과 건설적 사고로 나타났다. 전 등 $^{5)}$ 의 연구결과에서도 셀프리더십 자기존중, 자기보상, 리 허설, 목표설정, 자기비판, 건설적 사고 6개의 구성요인과 스 트레스 대처방식의 문제 중심 대처법, 소망사고 대처법 간에
는 모두 높은 상관관계가 있는 것과 같은 양상이다.

셀프 리더십과 스트레스 대처 척도간에 양의 상관관계가 나타났는데 전 등희 의 연구결과와 Folkman과 Lazarus ${ }^{14)}$ 도 같 은 결과이다. Christyn 등리 의 연구에서 셀프리더십과 심리요 인 중 접근적 대처에서 양의 상관관계가 나타난 것과 유사하 다.

즉 셀프 리더십이 높은 학생은 자신의 능력에 대한 확신이 높고 목표를 설정하고 스스로 실행에 옮겨보며 성공여부에 대한 자기비판을 하려는 반면, 스트레스 상황에 있어서는 긍 정적인 사고패턴으로 맞서는 특성이 나타난 것을 알 수 있다.

임상이라는 불확실한 환경은 스트레스를 유발하는데 장과 권 $^{24)}$ 은 스트레스 상황이 장기간 지속되면, 학생들의 경우 지적 능력이 약화되어 수학능력이 떨어지고 이러한 상황으로 부터의 도피가 결과적으로 학업포기를 가져올 수 있다고 하 였다.

하지만 셀프 리더십은 학습이나 교육을 통하여 발전하고 유지되는 개념흐으로 셀프 리더십이 낮은 집단에게 셀프 리더 십 훈련 프로그램을 투입시켜 임상 스트레스에 대처할 수 있는 심리요인적 특성을 향상시켜 전반적인 학업생활에 만족 도를 높일 수 있도록 해야 할 것이다.

즉 치위생과 학생들의 임상 실습에 대한 스트레스를 줄여 주고 스트레스에 잘 대처하게 하기 위해서는 임상실습 전이 나 초기에 셀프 리더십을 향상을 위한 구체적인 교육과정 개설, 운영 프로그램이 필요하다. 추후에 구체적으로 성별, 학년별, 임상실습 만족별 보다 구체적인 변인을 적절히 분석 하는 연구가 진행되어져야 할 것이다.

\section{결론}

본 연구는 대구 경북 4 개의 치위생(학)과 221명을 대상으로 셀프리더십이 임상실습 스트레스와 스트레스 대처 방식에 미치는 영향에 대해서 살펴보았고 다음과 같은 결론이 나타 났다.

첫째, 셀프 리더십이 높게 나타난 집단의 임상실습스트레 스 평균은 116.94였고, 셀프리더십 높은 집단의 스트레스 대 처는 평균 60.36이였고, 셀프리더십 낮은 집단의 평균은 57.20 이었으며 두 집단은 통계적으로 유의한 것으로 나타났 다.

둘째, 셀프 리더십의 하위 요소 중 임상실습 스트레스를 예측할 수 있는 요소는 자기기대, 리허설, 자기비판으로 나타 났으며, 셀프 리더십의 하위요소가 임상실습스트레스와의 관계를 예측할 수 있는 변수는 자기기대, 리허설, 자기비판이 었다. 
셋째, 셀프 리더십의 하위 요소 중 임상실습 스트레스 방안 을 예측할 수 있는 요소는 자기보상과 건설적 사고로 나타났 으며, 셀프 리더십의 하위요소가 스트레스 대처와의 관계를 예측할 수 있는 변수는 자기보상, 건설적 사고였다.

이상의 연구 결과에 의하면 치위생(학)과 학생들의 셀프 리더십은 임상실습 스트레스 및 대처에 영향을 미치지만 추 후 셀프 리더십에 영향을 미치는 다른 요인들과의 확대연구 가 필요하며 이를 토대로 셀프리더십 향상을 위한 프로그램 개발이 필요함을 제언한다.

\section{References}

1. Park NH. Personnel management. Seoul: Sumunsa; 2000: 25-33.

2. Lee JS. The relationship on nurses' self-leadership, job satisfaction and nursing performance[Master's Thesis]. Seoul: Univ. of Hanyang, 2010.

3. Manz CC, Sims HP. Business without bosses-how self-managing teams are building high-performing companice. New York: John Wiley \& Sons; 1995: 132-3.

4. Seomun GA. The relationship of self-leadership, job satisfaction, and prceived outcome in Nurses. J Korean Nurs Admi Acad Soc 2005; 11(1): 45-58.

5. Chun JY, Shin MS, Kim SK. Effect of self-leadership onstress coping in dental hygiene students. J Korean Soc Dent Hyg 2012; 12(2): 285-92.

6. Zimmermam BJ, Martinez-Pons M. Student difference in self-regulated learning relating grade, sex and giftedness to self-effiacacy and strategy use. J Educ psychol 1990; 82(1): 51-9.

7. Whang SJ. The relationship between clinical stress, self-efficacy, and self-esteem of nursing college students. J Korean acad soc nurs edu 2006; 12(2): 205-13.

8. Park MA. Self-leadership, stress on clinical practice, and stress coping styles in nursing students[Master's Thesis]. Daegu: Univ. of Kyungbook, 2009.

9. Manz C. Helping yourself and others to master self-leadership. Supervisory Management 1991; 36(11): 8-9.

10. Kim HS. The Relationship between teachers self - leader ship and job satisfaction at secondary schools[Master's Thesis]. Seoul: Univ. of Soongsil, 2003.

11. Shim HS. Status of clinical practice by dental hygiene student at Kwangju - Chonnam area colleges[Master's Thesis]. Gwangju: Univ. of Chosun, 2001.

12. Hwang SH. A study on the stressors experienced during clinical practice of dental hygiene students[Master's Thesis]. Seoul: Univ. of Donkook, 2004.

13. Bae JM, Jung EK, Yoo TW. Huh BL. A quick measurement of stressin outpatient clinic setting. Korean J Fam Medi 1992; 13(10): 809-20.

14. Folkman S, Lazarus R. If it changes it must be a process: study of emotion coping during three stage of a college examination. J Pers and Soc Psyc 1985; 48: 150-70.

15. Kim JH. Relations of perceived stress, cognitive set, and coping behaviors to depression[Doctor's thesis]. Seoul: Univ. of Seoul, 1987.

16. Park JY. A study on the relationship among character type A \& B ego-identity and stress coping[Master's Thesis]. Seoul: Univ. of Sookmyung Womens, 1995.

17. Nam YO, Jang JH. Kim SS. According to the clinical training, the study of stresses and coping ways of stresses. J Korean Soc Dent Hyg 2002; 2(2): 145-58.

18. Lee JH, Jeon ES. A study on causes of stress experienced by party students of dental hygiene department during clinical training. J Dent Hyg Sci 2009; 9(1): 137-43.

19. Yang NY, Moon SY. Relationship of self-leadership, stress and satisfaction in clinical practice of nursing students. $J$ korean Acad Nurs Admin 2011; 17(2): 216-25.

20. Neck CP, Stewart GW, Manz, OC. Thought self-leadership as a framework for enhancing the performance of performance appraises. J Appl Behav Sci 1995; 31(3): 278-302.

21. Kim NH. Super leader-ship. Seoul: Kyungmoonsa; 2002: 26-7.

22. Seomun GA, Chang SO, Cho KH, Kim IA, Lee SJ. The relation between self-leadership and outcome of nursing practice. $J$ Korean Acad Nurs Admin 2006; 12(1): 151-8.

23. Christyn LD, Mike S, Mary AS. The relationships between self-leadership and enhanced psycholocial, health, and work outcomes. J psychology 2001; 135(5): 469-85.

24. Jang SY, Kown SB. A research of stresses caused by extramural practices by hospital. J Korean Soc Dent Hyg 2011; 11(6): 913-22. 